\title{
BMJ Open Psychometric properties of the Chinese version of the WHOQOL-HIV BREF to assess quality of life among people living with HIV/AIDS: a cross- sectional study
}

Yaxin Zhu, ${ }^{1}$ Jie Liu, ${ }^{2}$ Bo Qu ${ }^{1}$

To cite: Zhu Y, Liu J, Qu B. Psychometric properties of the Chinese version of the WHOQOL-HIV BREF to assess quality of life among people living with HIV/AIDS: a crosssectional study. BMJ Open 2017;7:e016382. doi:10.1136/ bmjopen-2017-016382

- Prepublication history for this paper is available online. To view these files please visit the journal online (http://dx.doi org/10.1136/bmjopen-2017016382).

Received 11 February 2017 Revised 29 May 2017 Accepted 3 July 2017
CrossMark

${ }^{1}$ Department of Social Medicine, School of Public Health, China Medical University, Shenyang, China

${ }^{2}$ Department of Health Statistics, School of Public Health, China Medical University, Shenyang, China

Correspondence to

Dr Bo Qu; qub06666@163.com

\section{ABSTRACT}

Objectives This study aims to assess the psychometric properties of the Chinese version of the WHOQOL-HIV BREF.

Design Cross-sectional study.

Setting Centers for Disease Control and Prevention and infectious disease hospitals in three Chinese provinces.

Participants Sample of 1100 people living with HIV/AIDS (PLWHA).

Interventions We recruited 1100 PLWHA to evaluate their quality of life (QOL) using the WHOQOL-HIV BREF. Of these participants, 57 were randomly selected to repeat the QOL evaluation 2 weeks later.

Main outcome measures The reliability of the WHOQOLHIV BREF was assessed in terms of its internal consistency and test-retest reliability. The construct, concurrent, convergent, discriminant and known-group validity were also analysed. In addition, the factorial invariance across genders was assessed.

Results Cronbach's $\alpha$ coefficient for the overall scale was 0.93 . Except for the spirituality domain, which had an $\alpha$ below $0.70(0.66)$, the other five domains showed adequate internal consistency. The test-retest reliability revealed a statistically significant intraclass correlation coefficient of $0.72-0.82(p<0.001)$. Confirmatory factor analysis found that the six-domain structure produced an acceptable fit to the data. The instrument showed factorial invariance across gender groups. All domains were significantly correlated with the general items and the SF-36 $(p<0.01)$. The correlation coefficients were $>0.40$ $(r=0.40-0.67)$, except for the association between the spirituality domain and two general items (QOL: $r=0.33$; health status: $r=0.36)$. Subjects with lower CD4 counts had lower scores for all domains $(p<0.05)$. Symptomatic participants had significantly lower scores than asymptomatic participants on the physical, psychological and independence domains $(p<0.05)$.

Conclusions The WHOQOL-HIV BREF revealed good psychometric characteristics among Chinese PLWHA. These findings offer promising support for the use of the WHOQOL-HIV BREF as a measure of QOL among Chinese PLWHA and in cross-cultural comparative studies on QOL.
Strengths and limitations of this study

- This study offers promising support for the use of the WHOQOL-HIV BREF as a measure of quality of life (QOL) among Chinese people living with HIV/ AIDS (PLWHA) and in cross-cultural comparative studies on QOL.

- In our study, the tool's psychometric properties were analysed comprehensively.

- The study sample was recruited from three provinces encompassing the geographic diversity of northern, central and southern China.

- A longitudinal design might further measure the sensitivity of this instrument to changes in HIVrelated indicators such as CD4 count and HIV stage.

\section{INTRODUCTION}

The number of people living with HIV/AIDS (PLWHA) in China increased from 351709 in $2011^{1}$ to 577423 in $2015 .^{2}$ According to national data, as of February 2016, China had 591632 PLWHA. $^{3}$ Although the number of PLWHA is rising, the fatality rate for AIDS has decreased gradually since 2011 as the result of increasing availability of highly active antiretroviral therapy (HAART). ${ }^{1}$ The national epidemic data showed that the number of PLWHA receiving HAART increased from 295358 in $2014^{1}$ to 382139 in $2015 .^{2}$ The expanded use of HAART has increased the life expectancy of PLWHA. ${ }^{45}$

Since the advent of HAART, AIDS has progressed from an acute fatal infection into a manageable chronic disease. ${ }^{46}$ The evaluation of quality of life (QOL) has proven to be crucial in chronic disease treatment over the past decades. ${ }^{78}$ Moreover, because AIDS is an incurable disease, PLWHA will continue to suffer from the disease. $^{7}$ Thus, QOL assessments have recently become an essential element in 
AIDS care ${ }^{79-12}$ and improving the QOL of PLWHA has become a priority. ${ }^{13}$

In recent years, there has been much research on AIDS-related QOL. ${ }^{12-17}$ To better understand and evaluate the QOL of PLWHA, a reliable and valid measurement tool for QOL is important. ${ }^{711}$ Several instruments have been applied to assess the QOL of PLWHA such as the EQ-5D, ${ }^{15}$ SF-36, ${ }^{17}$ WHOQOL-HIV BREF, ${ }^{16}$ MOS-HIV ${ }^{14}$ AIDS-HAQ $^{18}$ and FAHI. ${ }^{19}$ Compared with generic questionnaires, AIDS-specific questionnaires have greater relevance and sensitivity. ${ }^{20}$ Reychler also suggested that disease-specific tools for assessing QOL are of particular relevance for patients infected with HIV. ${ }^{7}$ Some instruments have been developed within a single culture, particularly the Western culture. In addition, versions that have been translated into a different language often present less equality between the semantics and the concept, as demonstrated by their poorer psychometric characteristics. ${ }^{132122}$ Furthermore, previous studies have suggested that a cross-culturally valid QOL measure may be important for assessing health delivery in various cultural conditions. ${ }^{620}$

In 2003, the WHO developed the WHOQOL-HIV with 120 items (100 generic items and 20 HIV-related items). ${ }^{20}{ }^{23}$ The WHOQOL-HIV is a multidimensional instrument developed through a multinational collaboration. Furthermore, the instrument has been proven by a field test to be promising for assessing QOL in different cultural contexts. ${ }^{24}$ The Chinese version of the WHOQOL-HIV was developed by the research team from Sun Yat-sen University and China Academy of Chinese Medical Sciences. ${ }^{25}{ }^{26}$ The translation and cross-cultural adaptation of the original English version of the WHOQOL-HIV into Chinese were performed according to the method proposed by the WHO. The implementation of this method includes the following steps: forward translation, expert panel review, back translation, pretest and cognitive interviews and formulation of the final version. $^{25} 26$

The WHOQOL-HIV BREF is the short version of the WHOQOL-HIV and includes 31 items covering six domains. ${ }^{9}$ Additionally, it has been translated into different languages and has been proven to be reliable and valid..$^{91627}$ Although a few studies have applied this instrument to Chinese PLWHA, ${ }^{28}{ }^{29}$ its psychometric properties have not been fully assessed. This study was designed to assess the psychometric properties of the WHOQOL-HIV BREF among Chinese PLWHA. These findings may be of great importance for better understanding their QOL.

\section{MATERIALS AND METHODS \\ Ethics statement}

The ethical protocol was obtained from the Bioethics Advisory Commission of China Medical University. The investigators informed all participants about the purpose of the study and assured them before the research began that their privacy would be protected. All subjects provided written informed consent and voluntarily completed self-administered questionnaires.

\section{Study population and procedures}

A convenience sample was recruited from five cities (Shenyang, Dalian, Dandong, Zhengzhou and Ningbo) encompassing the geographic diversity of northern, central and southern China. Shenyang, Dalian and Dandong are three cities in the Liaoning province, which is situated in the northeast region of China. Zhengzhou is the capital of the Henan province in the central region of China. Ningbo is a city in the Zhejiang province, which is in the southern region of China. The inclusion criteria were that participants were 18 years or older, infected with HIV and without cognitive impairment. In total, 1201 PLWHA were recruited from local Centers for Disease Control and Prevention branches and infectious disease hospitals between February 2015 and January 2016. All subjects received a self-administered questionnaire and were compensated with 50 Chinese Yuan after completing the questionnaire. To ensure the quality of the questionnaire, specially trained investigators inspected the questionnaires after their completion and identified those that were missing answers or had more than one answer. If $>20 \%$ of the responses on one questionnaire were missing, the questionnaire was considered invalid. Questionnaires with valid responses were collected from 1100 participants total, and the valid response rate was $91.6 \%$. Shoukri et al suggested that the required sample size for a test-retest reliability study to estimate an intraclass correlation coefficient (ICC) of 0.8 and a $95 \%$ CI with a width of 0.2 (ie, the value of ICG is between 0.7 and 0.9 ) was $52 .{ }^{30}$ To examine the test-retest reliability, 57 participants were randomly selected to complete the QOL evaluation again 2 weeks later.

\section{Questionnaire}

The questionnaire comprised four sections including sociodemographic information, HIV-related characteristics, the WHOQOL-HIV BREF and the SF-36. The sociodemographic information included age, gender and work status. The HIV-related characteristics consisted of HIV stage, CD4 cell counts, mode of HIV transmission and time since diagnosis. As categorised by the clinically meaningful cut-off points, ${ }^{31} \mathrm{CD} 4$ cell counts were stratified into three groups. The HIV stage was divided into two groups: HIV-asymptomatic and HIV-symptomatic. ${ }^{132132}$

The WHOQOL-HIV BREF consisted of 31 items rated on a five-point Likert scale. Among the 31 items, 29 domain-specific items were used to measure individual QOL across six domains. The other two items were used to measure participants' perception of their general QOL and health status. The domain scores were calculated by multiplying the mean of all items within the domain by 4 . All domain scores ranged from 4 to 20 . 
Higher scores in each domain indicated higher QOL for that domain.

The SF-36 is a generic QOL measurement tool that has proven to be reliable and clinically valid for assessing the QOL of PLWHA. ${ }^{33-36}$ The SF-36 included 36 items covering eight domains, and the domain scores formed two summary scores ${ }^{7}$ : the physical component summary (PCS) and the mental component summary (MCS) scores, which ranged from 0 to $100 .^{37}{ }^{38}$ Higher scores indicated a better QOL. The reliability of this instrument in our study was satisfactory, with the $\alpha$ equal to 0.93 .

\section{Statistical analysis}

Missing data were replaced by a median. The mean, SD, skewness, kurtosis, floor effects and ceiling effects of each item and domain were computed. Floor or ceiling effects were significant if the percentage of subjects with the lowest or the highest score was $>20 \% .{ }^{27} 39$ A Cronbach's $\alpha$ coefficient of $\geq 0.70$ was considered to be an acceptable level of internal consistency. An ICC was applied for test-retest reliability, with an ICC $\geq 0.70$ indicating good test-retest reliability. ${ }^{40}$

To test the construct validity, a confirmatory factor analysis (CFA) was used. ${ }^{41}$ Goodness of fit was evaluated using the indexes including $\chi^{2}$, root mean square error of approximation (RMSEA), comparative fit index (CFI) and adjusted goodness-of-fit index (AGFI). An RMSEA value $<0.08$ and a CFI value $>0.90$ indicated a good fit. ${ }^{41}$ For AGFI, a value $>0.85$ was considered to be an adequate model fit. ${ }^{41}$ A multiple-group CFA analysis was conducted to investigate whether the WHOQOL-HIV BREF measured the same constructs across gender groups. First, we assessed the configural invariance. The next step involved assessing the metric invariance by examining if the factor loadings were the same across gender groups. Changes in CFI $(\Delta \mathrm{CFI} \leq 0.01)$ were used to demonstrate factorial invariance across groups. ${ }^{1342}$

Regarding concurrent validity, all domains were correlated with two general items (QOL and health status) and the SF-36. ${ }^{725}$ The scale's convergent and discriminant validity were tested by calculating item-domain Pearson's correlations. A correlation coefficient $>0.4$ for items and their respective domains was considered to be satisfactory. ${ }^{727}$ Items revealing correlations with their respective domains that were higher than those with other domains indicated good discriminant validity. ${ }^{43}$

Known-group validity was used to test how well the WHOQOL-HIV BREF discriminated among the subgroups of participants with regard to CD4 count and HIV stage. A multivariate analysis of variance was conducted to analyse the known-group validity. Post hoc tests were conducted to examine significant differences in domain scores among the three CD4 groups. It was hypothesised that HIV-symptomatic participants and PLWHA with lower CD4 counts would have significantly lower QOL domain scores. Values of $\mathrm{p}<0.05$
Table 1 Sociodemographic and HIV-related characteristics of the sample $(n=1100)$

\begin{tabular}{|c|c|c|}
\hline Characteristic & Number & Percentage (\%) \\
\hline \multicolumn{3}{|l|}{ Gender } \\
\hline Male & 965 & 87.7 \\
\hline Female & 135 & 12.3 \\
\hline \multicolumn{3}{|l|}{ Work status } \\
\hline Employed full time & 643 & 58.4 \\
\hline Employed part time & 420 & 38.2 \\
\hline Unemployed & 37 & 3.4 \\
\hline \multicolumn{3}{|l|}{ Marital status } \\
\hline Single & 450 & 40.9 \\
\hline Married & 443 & 40.3 \\
\hline Divorced/widowed & 207 & 18.8 \\
\hline \multicolumn{3}{|l|}{ Education level } \\
\hline Primary school or lower & 138 & 12.5 \\
\hline Junior high school & 331 & 30.1 \\
\hline Senior high school & 235 & 21.4 \\
\hline Junior college & 184 & 16.7 \\
\hline College or above & 212 & 19.3 \\
\hline \multicolumn{3}{|l|}{ Residence } \\
\hline Shenyang & 193 & 17.6 \\
\hline Dalian & 205 & 18.6 \\
\hline Dandong & 123 & 11.2 \\
\hline Zhengzhou & 381 & 34.6 \\
\hline Ningbo & 198 & 18.0 \\
\hline \multicolumn{3}{|l|}{ Monthly income (Yuan) } \\
\hline$<1000$ & 222 & 20.2 \\
\hline $1001-2000$ & 256 & 23.3 \\
\hline $2001-3000$ & 314 & 28.5 \\
\hline$\geq 3001$ & 308 & 28.0 \\
\hline \multicolumn{3}{|l|}{ HIV stage } \\
\hline Symptomatic & 409 & 37.2 \\
\hline Asymptomatic & 691 & 62.8 \\
\hline \multicolumn{3}{|l|}{ CD4 count (cells/mm³) } \\
\hline$<200$ & 99 & 9.0 \\
\hline 200-499 & 642 & 58.4 \\
\hline$\geq 500$ & 359 & 32.6 \\
\hline \multicolumn{3}{|l|}{ Mode of HIV transmission } \\
\hline Male homosexual sex & 713 & 64.8 \\
\hline Heterosexual sex & 210 & 19.1 \\
\hline Drug abuse & 11 & 1.0 \\
\hline Blood product & 112 & 10.2 \\
\hline Unknown & 54 & 4.9 \\
\hline
\end{tabular}


Open Access

Table 2 Descriptive statistics of the WHOQOL-HIV BREF $(n=1100)$

\begin{tabular}{|c|c|c|c|c|c|}
\hline Domain or item & Mean \pm SD & Skewness & Kurtosis & Floor (\%) & Ceiling (\%) \\
\hline General QOL & $3.27 \pm 0.92$ & -0.18 & 0.5 & 5.0 & 9.8 \\
\hline General health status & $3.10 \pm 0.94$ & -0.09 & -0.09 & 5.2 & 6.6 \\
\hline Physical & $13.76 \pm 2.94$ & -0.19 & -0.25 & 0.1 & 1.5 \\
\hline Pain and discomfort & $4.01 \pm 0.99$ & -0.98 & 0.46 & 1.8 & 35.9 \\
\hline Symptoms of PLWHA & $3.48 \pm 1.08$ & -0.49 & -0.64 & 3.9 & 15.3 \\
\hline Energy and fatigue & $3.19 \pm 1.19$ & -0.02 & -0.91 & 7.9 & 17.6 \\
\hline Sleep and rest & $3.08 \pm 1.04$ & -0.19 & -0.33 & 8.7 & 8.3 \\
\hline Psychological & $12.16 \pm 2.81$ & 0.26 & -0.16 & 0.1 & 0.8 \\
\hline Positive feelings & $2.83 \pm 1.09$ & 0.04 & -0.71 & 12.5 & 5.9 \\
\hline Cognition & $2.90 \pm 1.00$ & 0.01 & -0.47 & 8.3 & 5.0 \\
\hline Body image and appearance & $3.07 \pm 1.11$ & 0.10 & -0.50 & 8.1 & 13.3 \\
\hline Self-esteem & $3.17 \pm 0.98$ & -0.25 & -0.18 & 5.8 & 7.4 \\
\hline Negative feelings & $3.24 \pm 0.99$ & 0.03 & -0.25 & 4.0 & 12.5 \\
\hline Independence & $13.19 \pm 2.82$ & -0.13 & 0.17 & 0.2 & 1.6 \\
\hline Dependence on medication/treatment & $3.02 \pm 1.12$ & 0.07 & -0.78 & 8.3 & 10.7 \\
\hline Mobility & $3.57 \pm 0.96$ & -0.42 & 0.13 & 3.5 & 17.2 \\
\hline Activities of living & $3.32 \pm 0.91$ & -0.29 & 0.20 & 3.9 & 8.9 \\
\hline Working capacity & $3.28 \pm 0.96$ & -0.36 & 0.03 & 5.4 & 8.6 \\
\hline Social relationships & $12.15 \pm 2.91$ & -0.02 & 0.16 & 0.9 & 0.9 \\
\hline Social inclusion & $2.60 \pm 1.20$ & 0.28 & -0.81 & 22.4 & 7.2 \\
\hline Personal relationships & $3.27 \pm 0.90$ & -0.36 & 0.27 & 4.4 & 6.8 \\
\hline Sex life & $2.96 \pm 0.94$ & -0.23 & 0.04 & 8.5 & 4.2 \\
\hline Social support & $3.31 \pm 0.91$ & -0.35 & 0.28 & 4.4 & 8.3 \\
\hline Environment & $12.05 \pm 2.88$ & 0.01 & -0.05 & 0.5 & 0.5 \\
\hline Physical safety and security & $2.85 \pm 1.03$ & -0.01 & -0.53 & 10.5 & 4.9 \\
\hline Home environment & $2.97 \pm 1.00$ & -0.09 & -0.24 & 8.5 & 6.0 \\
\hline Financial resources & $2.54 \pm 1.19$ & 0.33 & -0.70 & 24.2 & 7.1 \\
\hline Opportunities for information and skills & $2.95 \pm 1.13$ & 0.03 & -0.72 & 11.0 & 9.2 \\
\hline Opportunities for recreation and leisure & $2.89 \pm 1.21$ & 0.11 & -0.89 & 14.2 & 11.0 \\
\hline Physical environment & $3.25 \pm 0.94$ & -0.37 & 0.08 & 5.3 & 7.4 \\
\hline Access to health and social care & $3.38 \pm 1.00$ & -0.48 & 0.06 & 6.0 & 11.5 \\
\hline Transport & $3.26 \pm 0.94$ & -0.31 & 0.08 & 4.9 & 8.2 \\
\hline Spirituality & $13.07 \pm 3.44$ & -0.32 & -0.41 & 0.8 & 1.0 \\
\hline Spiritual & $2.89 \pm 1.14$ & -0.01 & -0.78 & 13.4 & 7.9 \\
\hline Forgiveness & $3.32 \pm 1.23$ & -0.22 & -0.84 & 7.5 & 20.2 \\
\hline Fear of the future & $3.39 \pm 1.22$ & -0.43 & -0.83 & 8.4 & 19.2 \\
\hline Death and dying & $3.46 \pm 1.30$ & -0.51 & -0.87 & 11.0 & 25.1 \\
\hline
\end{tabular}

PLWHA, people living with HIV/AIDS; QOL, quality of life.

were considered to be statistically significant. SPSS 19.0 and LISREL 8.5 software for Windows were used.

\section{RESULTS}

Basic characteristics of the study sample

The mean age of the 1100 participants was 39.62 years, with an SD of 12.73. Most of the participants were male (965, 87.7\%). Of the 1100 participants, $713(64.8 \%)$ were infected with HIV through male homosexual sex. A total 
Table 3 Internal consistency and test-retest reliability of the WHOQOL-HIV BREF

\begin{tabular}{lll}
\hline & $\begin{array}{l}\text { Cronbach's } \\
\boldsymbol{\alpha} \text { coefficient } \\
(\mathbf{n = 1 1 0 0 )}\end{array}$ & ICC (95\% CI) (n=57) \\
\hline Domain & 0.71 & $0.76(0.63-0.85)^{\star \star *}$ \\
\hline Physical & 0.70 & $0.73(0.57-0.83)^{\star \star \star}$ \\
\hline Psychological & 0.85 & $0.82(0.71-0.89)^{\star \star \star}$ \\
Independence & 0.71 & $0.80(0.69-0.88)^{\star \star \star}$ \\
Social relationships & 0.83 & $0.74(0.59-0.84)^{\star \star \star}$ \\
\hline Environment & 0.66 & $0.72(0.56-0.82)^{\star \star \star}$ \\
\hline Spirituality & &
\end{tabular}

${ }^{*} \mathrm{p}<0.001$.

ICC, intraclass correlation coefficient.

of $691(62.8 \%)$ were asymptomatic. The average time since diagnosis was 3.91 years $(\mathrm{SD}=2.85)$. The sociodemographic and HIV-related characteristics are shown in table 1 .

\section{Score distributions}

The descriptive statistics of each item and domain are displayed in table 2. The skewness and kurtosis coefficients of all items and domains ranged from -1.00 to 1.00 , which were acceptable. No significant floor or ceiling effects were found in the six domains. However, the item measuring pain and discomfort showed a significant ceiling effect $(35.9 \%)$, and floor effects were detected in the items measuring social inclusion $(22.4 \%)$ and financial resources $(24.2 \%)$. Across domains, the physical domain had the highest score (13.76 \pm 2.94$)$, and the environment domain score was the lowest $(12.05 \pm 2.88)$.

\section{Reliability}

The internal consistency was excellent, with an overall Cronbach's $\alpha$ coefficient of 0.93 . The spirituality domain had an $\alpha$ of 0.66 , which was slightly below the cut-off value of 0.70 for acceptable internal consistency. The other five domains were confirmed to have adequate internal consistency. The test-retest reliability showed a statistically significant ICC for all domains. The test-retest values were good, with the ICC ranging from 0.72 (spirituality domain) to 0.82 (independence domain) $(\mathrm{p}<0.001)$. The results are reported in table 3 .

\section{Construct validity}

The CFA results showed that the six-domain structure of the WHOQOL-HIV BREF produced an acceptable fit to the data $\left(\chi^{2}=5662.69, \mathrm{df}=362, \mathrm{p}<0.001 ; \mathrm{CFI}=0.81\right.$; RMSEA $=0.07$ [90\% CI: 0.04 to 0.08]; AGFI=0.87). Except for the item measuring spiritual, the factor load of each item with its respective domain was acceptable, ranging from 0.35 to 0.89 , as shown in figure 1 .

\section{Concurrent validity}

The correlation coefficients of all domains with the two general measures (general QOL and general health status) and two component summary scores of the SF-36
(PCS and MCS) are displayed in table 4. All domains correlated with both general QOL and health status significantly $(\mathrm{p}<0.01)$. The correlation coefficients were $>0.40(\mathrm{r}=0.44-0.57)$, with the exception of the association between the spirituality domain and the two items (QOL: $r=0.33$; health status: $r=0.36$ ). In addition, the coefficients between all domains and two component summary scores were $>0.40(\mathrm{r}=0.40-0.67, \mathrm{p}<0.01)$. Generally, these results indicated satisfactory concurrent validity of the WHOQOL-HIV BREF.

\section{Convergent and discriminant validity}

Concerning item-domain correlations, each item correlated with its respective domain score, and $\mathrm{r}$ coefficients ranged from 0.47 to $0.84(\mathrm{p}<0.01)$. With regard to discriminant validity, the majority of items revealed a higher correlation with their respective domains than with other domains $(p<0.01)$. However, the item 'spiritual' showed a higher correlation with the psychological domain $(\mathrm{r}=0.65, \mathrm{p}<0.01)$ than with its respective (ie, spirituality) domain $(\mathrm{r}=0.50$, $\mathrm{p}<0.01)$. Generally, convergent and discriminant validity were considered to be good (table 5).

\section{Known-group validity}

A significant multivariate effect was found for CD4 counts (Wilks' $\lambda=0.956, F_{12,2184}=4.12, \mathrm{p}<0.001, \eta_{\mathrm{P}}^{2}=0.022$ ). Subsequent univariate $F$-tests, which are shown in table 6 , indicated that all six domains contributed to the multivariate effect. Among the three groups divided by CD4 count, the mean scores for the six domains in group $\mathrm{C}$ (CD4 count $\geq 500$ cells $/ \mathrm{mm}^{3}$ ) were the highest, and the subjects with a CD 4 count $<200$ cells $/ \mathrm{mm}^{3}$ showed the lowest scores $(\mathrm{p}<0.05)$.

\section{DISCUSSION}

Our results suggested that the WHOQOL-HIV BREF is reliable and valid for Chinese PLWHA. The skewness and kurtosis coefficients of all items and domains were in the acceptable range $(-1.00$ to 1.00$)$, which was a similar result to previous studies conducted among Taiwanese patients infected with HIV and ageing Portuguese patients with HIV. ${ }^{64}$ In line with other studies, ${ }^{64}$ the ceiling effect of the item measuring pain and discomfort was above the accepted threshold of $20 \%$. It is likely that the ceiling effect is dependent on population distribution. ${ }^{44}$ Although previous studies have not reported the floor effects of the WHOQOL-HIV BREF, ${ }^{6} 72144$ we detected floor effects for two items (social inclusion and financial resources).

Except for the spirituality domain, all domains showed satisfactory reliability. The results are consistent with other reliability studies in Malaysia, Portugal and Ethiopia. ${ }^{2144-46}$ Saddki and Pereira suggested that the content and size of the spirituality domain could result in lower reliability. ${ }^{214}$ Tesfaye et al noted that the limitations of the spirituality domain in this instrument should be acknowledged. ${ }^{46}$ Chandra also indicated that the 


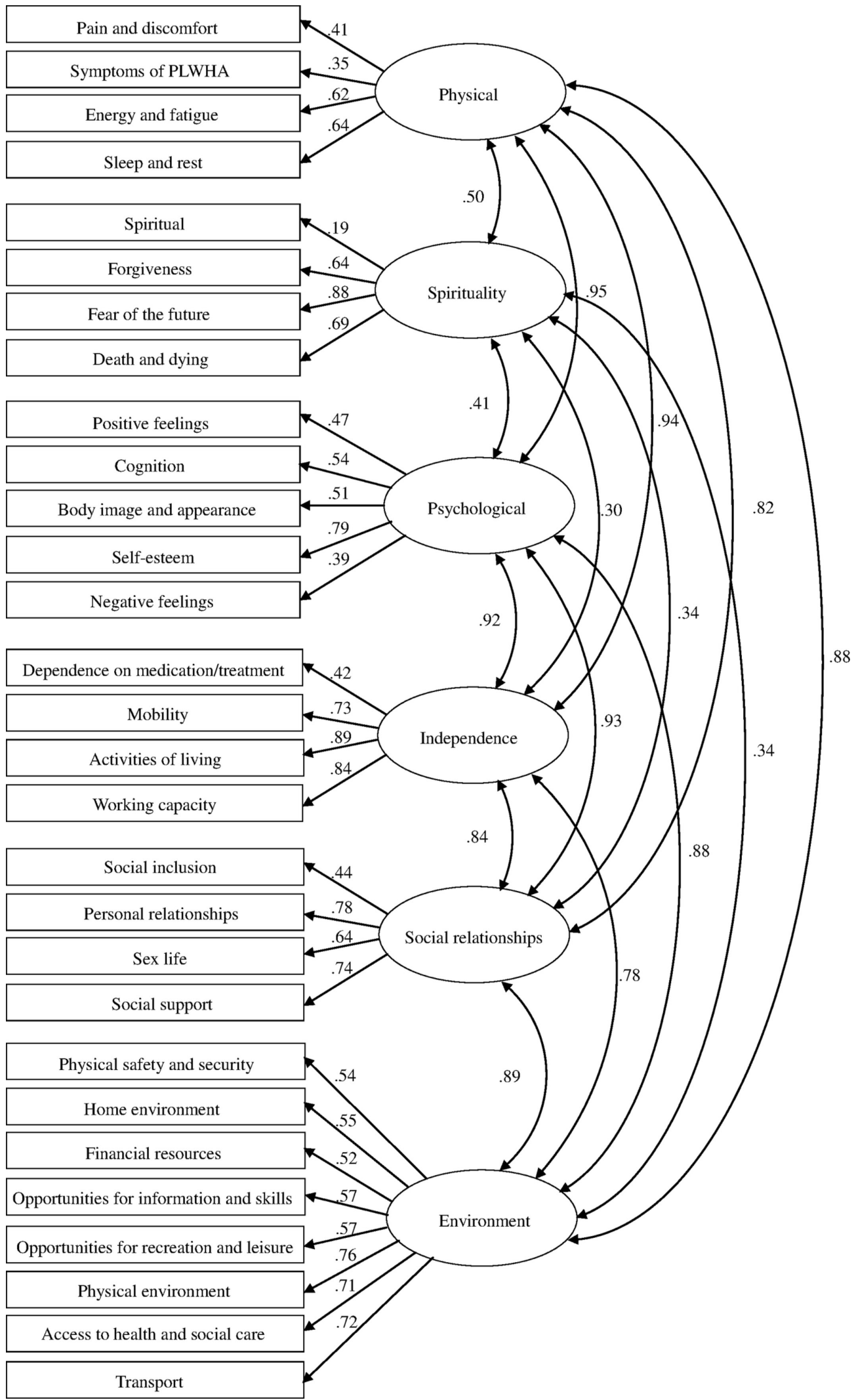

Figure 1 The structure of the Chinese version of the WHOQOL-HIV BREF based on confirmatory factor analysis. The sixfactor structure of the WHOQOL-HIV BREF was tested across gender groups to evaluate factorial invariance. The results of configural invariance showed the invariance of the factor structure across gender groups: $\chi^{2}=6982.87, p<0.001, C F I=0.808$ and RMSEA $=0.072$. The findings for metric invariance showed that the factor loadings were the same across gender groups: $\chi^{2}=6960.17, p<0.001, \mathrm{CFI}=0.809$ and RMSEA $=0.073$. In addition, the change of CFI was 0.001 , which was lower than 0.01. The Chinese version of the WHOQOL-HIV BREF showed factorial invariance for PLWHA across gender groups. CFI, comparative fit index; PLWHA, people living with HIV/AIDS; RMSEA, root mean square error of approximation. 
Table 4 Concurrent validity of the WHOQOL-HIV BREF $(n=1100)$

\begin{tabular}{|c|c|c|c|c|}
\hline \multirow[b]{2}{*}{ Domain } & \multicolumn{4}{|c|}{ Correlation coefficient } \\
\hline & General QOL & General health status & PCS & MCS \\
\hline Physical & $0.56^{\star \star \star}$ & $0.54^{\star \star \star}$ & $0.67^{\star \star \star}$ & $0.66^{\star \star \star}$ \\
\hline Psychological & $0.54^{\star * \star}$ & $0.50^{\star * *}$ & $0.55^{\star \star \star}$ & $0.62^{\star \star \star}$ \\
\hline Independence & $0.57^{\star \star \star}$ & $0.53^{\star \star *}$ & $0.63^{* * *}$ & $0.61^{\star \star *}$ \\
\hline Social relationships & $0.47^{\star \star \star}$ & $0.44^{\star \star *}$ & $0.46^{\star \star \star}$ & $0.49^{\star \star \star}$ \\
\hline Environment & $0.53^{\star * \star}$ & $0.46^{\star * *}$ & $0.49^{* * *}$ & $0.52^{* * *}$ \\
\hline Spirituality & $0.33^{\star \star \star}$ & $0.36^{\star \star \star}$ & $0.40^{\star \star \star}$ & $0.49^{\star \star \star}$ \\
\hline
\end{tabular}

${ }^{* \star *} p<0.01$; MCS, mental component summary; PCS, physical component summary; QOL, quality of life.

spirituality domain in the WHOQOL-HIV BREF may be inadequate, and he suggested that having more items in the spirituality domain on the WHOQOL-HIV could increase sensitivity in this regard. ${ }^{47}$ We also found that the WHOQOL-HIV BREF has satisfactory test-retest reliability, which is consistent with the French version. ${ }^{7}$

The results of the CFA suggested that the original six-domain structure could provide a generally good fit for our study data, which is consistent with other studies using the WHOQOL-HIV BREF. ${ }^{9}{ }^{44}{ }^{46}$ However, the exploratory factor analysis (EFA) of the WHOQOL-HIV BREF conducted with Portuguese and Malaysian patients positive for HIV yielded a five-factor model, which contradicts the original six-domain model. ${ }^{21}{ }^{45}$ In addition to the EFA, Canavarro also conducted a CFA of the WHOQOL-HIV BREF and found that both the five-factor model and the original six-domain model fit relatively well. ${ }^{45}$ Moreover, Peltzer's results from a multivariate logistic regression showed that four domains (psychological, independence, environment and spirituality) were major predictors of overall QOL. ${ }^{48}$ Some studies have suggested that the original model fit would improve if some items were modified. ${ }^{45}{ }^{46}$ The factor load of the spiritual item with its respective domain (ie, 'Spirituality' domain) was 0.11 , which was much lower than others. As previously suggested, we reassigned this item to the 'Psychological' domain in our study and then assessed the construct validity again. The result showed that the factor load of the spiritual item with the 'Psychological' domain was 0.67 , and the model fit also improved, with $\mathrm{CFI}=0.87$, RMSEA $=0.07$ (90\% CI: 0.05 to 0.08 ) and AGFI $=0.89$.

Concurrent validity was demonstrated by the significant correlations between two general items and the SF-36. All domains correlated significantly with self-perceived general QOL $(\mathrm{r}=0.33-0.57, \mathrm{p}<0.01)$, health status $(\mathrm{r}=0.36-0.54, \mathrm{p}<0.01)$ and two component summary scores of the SF-36 $(\mathrm{r}=0.40-0.67, \mathrm{p}<0.01)$. The correlation coefficient was comparable to that reported in the validation study of the Malay version ${ }^{21}$ but was somewhat lower than the results of other validation studies of the WHOQOL-HIV BREF. ${ }^{74}$ It was noted that the spirituality domain showed the lowest correlation with the general QOL $(r=0.33, p<0.01)$ and health status $(r=0.36$, $\mathrm{p}<0.01$ ). This finding is consistent with results found by Saddki. ${ }^{21}$ More specifically, regarding the findings related to discriminant validity, the item measuring spirituality showed a higher correlation with the psychological domain $(\mathrm{r}=0.65, \mathrm{p}<0.01)$ than with its initially assigned domain (ie, spirituality; $r=0.50, \mathrm{p}<0.01$ ). Canavarro also found this correlation in the European Portuguese version, and he suggested that this item should be modified or reassigned to the psychological domain. ${ }^{45}$

Demonstrating its known-group validity, the WHOQOL-HIV BREF discriminated between the CD4 count groups $(\mathrm{p}<0.05)$, which is consistent with previous studies. ${ }^{45}$ We also observed that the subjects with higher CD4 counts reported better QOL. This was also observed by other researchers. ${ }^{47}{ }^{48}$ However, Pereira indicated that

Table 5 Convergent and discriminant validity of the WHOQOL-HIV BREF $(n=1100)$

\begin{tabular}{|c|c|c|c|c|c|c|}
\hline \multirow[b]{2}{*}{ Domain } & \multicolumn{2}{|c|}{ Correlation coefficient range } & \multicolumn{2}{|c|}{ Convergent validity } & \multicolumn{2}{|c|}{ Discriminant validity } \\
\hline & $\begin{array}{l}\text { Convergent } \\
\text { validity }\end{array}$ & $\begin{array}{l}\text { Discriminant } \\
\text { validity }\end{array}$ & Success/total & Percentage (\%) & Success/total & Percentage (\%) \\
\hline Psychological & $0.57-0.73^{\star \star \star}$ & $0.15-0.67^{\star \star \star}$ & $5 / 5$ & 100 & $5 / 5$ & 100 \\
\hline Independence & $0.47-0.84^{\star * *}$ & $0.02-0.68^{\star * *}$ & $4 / 4$ & 100 & $4 / 4$ & 100 \\
\hline Spirituality & $0.50-0.84^{\star \star \star}$ & $0.14-0.65^{\star \star \star}$ & $4 / 4$ & 100 & $3 / 4$ & 75 \\
\hline
\end{tabular}

${ }^{* * *} \mathrm{p}<0.01$. 
Table 6 Known-group validity for subgroups of participants by CD4 count $(n=1100)$

\begin{tabular}{|c|c|c|c|c|c|}
\hline Domain & $\begin{array}{l}\text { Group A (<200 cells/ } \\
\left.\mathrm{mm}^{3}\right)\end{array}$ & $\begin{array}{l}\text { Group B (200-499 cells/ } \\
\left.\mathrm{mm}^{3}\right)\end{array}$ & $\begin{array}{l}\text { Group C ( } \geq 500 \text { cells/ } \\
\left.\mathrm{mm}^{3}\right)\end{array}$ & $F$ & $\eta_{\mathrm{P}}^{2}$ \\
\hline Physical $\ddagger \S$ & $12.63 \pm 2.56$ & $13.63 \pm 2.97$ & $14.30 \pm 2.87$ & $14.34^{* * *}$ & 0.025 \\
\hline Psychological $† \ddagger \S$ & $11.01 \pm 2.29$ & $12.05 \pm 2.75$ & $12.68 \pm 2.93$ & $15.24^{* * t *}$ & 0.027 \\
\hline Social relationships $\downarrow \ddagger \S$ & $11.32 \pm 2.32$ & $11.99 \pm 2.92$ & $12.65 \pm 2.96$ & $10.43^{* * *}$ & 0.019 \\
\hline Independence†‡§ & $12.09 \pm 2.50$ & $13.03 \pm 2.86$ & $13.77 \pm 2.72$ & $16.50^{* * *}$ & 0.029 \\
\hline Environment\$§ & $11.49 \pm 2.33$ & $11.93 \pm 2.81$ & $12.40 \pm 3.10$ & $5.09^{* *}$ & 0.009 \\
\hline Spirituality§ & $12.39 \pm 2.86$ & $12.98 \pm 3.46$ & $13.40 \pm 3.51$ & $3.81^{*}$ & 0.007 \\
\hline
\end{tabular}

${ }^{*}$ Group $\mathrm{A}$ is significantly different from group $\mathrm{C}$. $\dagger \mathrm{p}<0.05 ;{ }^{* \star} \mathrm{p}<0.01 ;{ }^{* \star *} \mathrm{p}<0.001$.

$\ddagger$ Group $A$ is significantly different from group $B$.

§Group B is significantly different from group $C$.

IRegarding HIV stage, the multivariate effect was also significant (Wilks' $\lambda=0.924, F_{6,1093}=6.92, p<0.001, \eta_{\mathrm{P}}^{2}=0.076$ ), and follow-up tests indicated that the symptomatic participants had significantly lower scores than asymptomatic participants in the physical (13.54 \pm 3.03$)$, psychological (11.69 \pm 2.78$)$ and independence $(12.75 \pm 2.73)$ domains $(p<0.05$, see table 7$)$.

Table 7 Known-group validity for subgroups of participants by HIV stage $(n=1100)$

\begin{tabular}{lllcc}
\hline Domain & Symptomatic & Asymptomatic & $\boldsymbol{F}$ & $\eta_{\mathrm{P}}^{2}$ \\
\hline Physical & $13.54 \pm 3.03$ & $14.10 \pm 3.00$ & $4.11^{* *}$ & 0.008 \\
Psychological & $11.69 \pm 2.78$ & $12.70 \pm 2.81$ & $15.43^{* *}$ & 0.029 \\
Social relationships & $12.02 \pm 3.03$ & $12.42 \pm 2.77$ & 2.39 & 0.005 \\
Independence & $12.75 \pm 2.73$ & $13.56 \pm 2.78$ & $10.28^{* *}$ & 0.020 \\
Environment & $12.01 \pm 2.87$ & $12.42 \pm 2.85$ & 2.49 & 0.005 \\
Spirituality & $13.62 \pm 3.17$ & $13.07 \pm 3.58$ & 3.07 & 0.006 \\
\hline
\end{tabular}

${ }^{*} \mathrm{p}<0.05 ;{ }^{* *} \mathrm{p}<0.01 ;{ }^{* \star *} \mathrm{p}<0.001$.

domain scores were not significantly different across CD4 counts among ageing adults with HIV, and he implied that associations between biological markers and QOL may conflict. ${ }^{44}$ Another result was that the physical, psychological and independence domains discriminated relatively well between symptomatic and asymptomatic PLWHA $(\mathrm{p}<0.05)$. A multinational pilot study also showed that the physical and independence domains discriminated better than other domains between different stages of HIV disease progression. ${ }^{9}$ The failure of the spirituality domain to discriminate between PLWHA in two HIV stages may imply that although AIDS is now a non-fatal disease, the extent of suffering from the related emotional distress remains similar at different HIV stages. ${ }^{21}$ A previous study suggested that, due to problems with understanding, the "safety and security' item could not discriminate between symptomatic and asymptomatic PLWHA. ${ }^{46}$ This may partly explain the failure of the environment domain. The social relationships domain may face the same problems, and further studies are needed to clarify this point. Other studies have found that the WHOQOL-HIV BREF has good validity with respect to disease stage, and asymptomatic PLWHA have better QOL than do symptomatic subjects. ${ }^{15} 49$

There are limitations to this study that should be acknowledged. First, potential limitations are introduced by convenience sampling and self-administration of an assessment. All subjects were enrolled by convenience, and the self-administered assessment resulted in the exclusion of PLWHA with difficulty in reading, which may have made the sample not representative of Chinese PLWHA. Second, various methods of administering the questionnaire such as self-administered and interviewer-administered methods should have been applied to assess its technical validity. Third, the difference of mean QOL between CD4 count subgroups is small but statistically significant. A longitudinal design might further measure the sensitivity of this instrument to changes in HIV-related indicators such as CD4 count and HIV stage; this improved sensitivity could clarify whether the difference is clinically meaningful. In addition, the percentage of missing values per item ranged from $0.2 \%$ to $2.3 \%$. Missing values were imputed as the median score of those who answered the item. As a result, the estimated variances underestimated the variances of the underlying distributions. Furthermore, there may be some problems in the spirituality domain, and the next step of our research should be to assess the psychometric properties again after translating and modifying this domain. 


\section{CONCLUSIONS}

The WHOQOL-HIV BREF revealed good psychometric characteristics among Chinese PLWHA. These findings offer promising support for the use of the WHOQOL-HIV BREF as a measure of QOL among Chinese PLWHA and in cross-cultural comparative studies on QOL. Further studies on the spirituality domain should be conducted to ensure the effectiveness of the WHOQOL-HIV BREF.

Acknowledgements The authors wish to thank Shijie Liang, Xia Min, and Li Wang for help with sample collection. We thank all the PLWHA participants in this study.

Contributors $\mathrm{BQ}$ designed the research. $\mathrm{YZ}$ and $\mathrm{JL}$ participated in the data collection and analysis. YZ wrote the manuscript. All authors read and approved the final manuscript.

Funding This work was supported by National Natural Science Foundation of China, grant numbers 71473269 and 71673301 . The funders had no role in study design, data collection and analysis, decision to publish or preparation of the manuscript.

Competing interests None declared.

Ethics approval Ethical protocol was obtained from the Bioethics Advisory Commission of China Medical University. All of the subjects provided written informed consent and voluntarily completed self-administered questionnaires.

Provenance and peer review Not commissioned; externally peer reviewed.

Data sharing statement All data from the current study were reported in the manuscript. Participant-level data are not publicly available due to ethical and legal obligations to the participants in the study. Data are available upon request to the corresponding author and with permission of the local ethics committee.

Open Access This is an Open Access article distributed in accordance with the Creative Commons Attribution Non Commercial (CC BY-NC 4.0) license, which permits others to distribute, remix, adapt, build upon this work non-commercially, and license their derivative works on different terms, provided the original work is properly cited and the use is non-commercial. See: http://creativecommons.org/ licenses/by-nc/4.0/

(C) Article author(s) (or their employer(s) unless otherwise stated in the text of the article) 2017. All rights reserved. No commercial use is permitted unless otherwise expressly granted.

\section{REFERENCES}

1. National Health and Family Planning Commission of the People's Republic of China. 2015 China AIDS Response Progress Report. 2015 http://www.aidsdatahub.org/sites/default/files/publication/ China narrative report 2015.pdf (accessed 16 Jan 2016).

2. NCAIDS, NCSTD, China CDC. Update on the AIDS/STD epidemic in China and main response in control and prevention in December 2015. Chin J AIDS STD 2016;22:69.

3. NCAIDS, NCSTD, China CDC. Update on the AIDS/STD epidemic in China and main response in control and prevention in February, 2016. Chin J AIDS STD 2016;22:223.

4. Mutabazi-Mwesigire D, Seeley J, Martin F, et al. Perceptions of quality of life among Ugandan patients living with HIV: a qualitative study. BMC Public Health 2014;14:343.

5. Mwesigire DM, Martin F, Seeley J, et al. Relationship between CD4 count and quality of life over time among HIV patients in Uganda: a cohort study. Health Qual Life Outcomes 2015;13:144.

6. Hsiung PC, Fang CT, Wu CH, et al. Validation of the WHOQOLHIV BREF among HIV-infected patients in Taiwan. AIDS Care 2011;23:1035-42.

7. Reychler G, Caty G, Vincent A, et al. Validation of the French version of the World Health Organization quality of life HIV instrument. PLOS One 2013;8:e73180.

8. Alonso J, Ferrer M, Gandek B, et al . Health-related quality of life associated with chronic conditions in eight countries: results from the International Quality of Life Assessment (IQOLA) Project. Qual Life Res 2004;13:283-98.

9. O'Connell KA, Skevington SM. An international quality of life instrument to assess wellbeing in adults who are HIV-positive: a short form of the WHOQOL-HIV (31 items). AIDS Behav 2012;16:452-60.
10. Starace F, Cafaro L, Abrescia N, et al. Quality of life assessment in HIV-positive persons: application and validation of the WHOQOLHIV, Italian version. AIDS Care 2002;14:405-15.

11. Wu AW. Quality of life assessment comes of age in the era of highly active antiretroviral therapy. AIDS 2000;14:1449-51.

12. Weinfurt KP, Willke RJ, Glick HA, et al. Relationship between CD4 count, viral burden, and quality of life over time in HIV-1-infected patients. Med Care 2000;38:404-10.

13. Skevington SM, Norweg S, Standage M. Predicting quality of life for people living with HIV: international evidence from seven cultures. AIDS Care 2010;22:614-22.

14. Mutabazi-Mwesigire D, Katamba A, Martin F, et al. Factors that affect quality of life among people living with HIV attending an urban clinic in Uganda: a cohort study. PLoS One 2015;10:e0126810.

15. Nglazi MD, West SJ, Dave JA, et al. Quality of life in individuals living with HIV/AIDS attending a public sector antiretroviral service in Cape Town, South Africa. BMC Public Health 2014;14:676.

16. Nobre N, Pereira M, Sutinen J, et al. Quality of life of people living with HIV/AIDS: a cross-country comparison study of Finland and Portugal. AIDS Care 2016;28:873-7.

17. Balderson $B H$, Grothaus L, Harrison RG, et al. Chronic illness burden and quality of life in an aging HIV population. AIDS Care 2013;25:451-8

18. Lubeck DP, Fries JF. Assessment of quality of life in early stage HIVinfected persons: data from the AIDS Time-oriented Health Outcome Study (ATHOS). Qual Life Res 1997;6:494-506.

19. Peterman AH, Cella D, Mo F, et al. Psychometric validation of the revised Functional Assessment of Human immunodeficiency virus Infection (FAHI) quality of life instrument. Qual Life Res 1997;6:572-84.

20. WHOQOL (World Health Organization's Quality of Life Instrument) HIV Group. Initial steps to developing the World Health Organization's Quality of Life Instrument (WHOQOL) module for international assessment in HIV/AIDS. AIDS Care 2003:15:347-57.

21. Saddki N, Noor MM, Norbanee TH, et al. Validity and reliability of the Malay version of WHOQOL-HIV BREF in patients with HIV infection. AIDS Care 2009;21:1271-8.

22. Bowden A, Fox-Rushby JA. A systematic and critical review of the process of translation and adaptation of generic health-related quality of life measures in Africa, Asia, Eastern Europe, the Middle East, South America. Soc Sci Med 2003;57:1289-306.

23. O'Connell K, Skevington S, Saxena S. Preliminary development of the World Health Organsiation's Quality of Life HIV instrument (WHOQOL-HIV): analysis of the pilot version. Soc Sci Med 2003:57:1259-75.

24. WHOQOL HIV Group. WHOQOL-HIV for quality of life assessment among people living with HIV and AIDS: results from the field test. AIDS Care 2004;16:882-9.

25. Cai NQ, Kang J, DI X, et al. Qualitative interview on modification of WHOQOL-HIV. J Tra Chin Med 2012:53:839-42.

26. Liu WM, Ly H, Wang J, et al. The introduce and usage of WHOQOLHIV BREF instrument in chinese. Chin J Info Tradit Chin 2009;16:1-2.

27. Fu TS, Tuan YC, Yen MY, et al. Psychometric properties of the World Health Organization Quality of Life Assessment-Brief in methadone patients: a validation study in Northern Taiwan. Harm Reduct $J$ 2013;10:37.

28. Ming Z, Prybylski D, Cheng F, et al. Two-year prospective cohort study on quality of life outcomes among people living with HIV after initiation of antiretroviral therapy in Guangxi, China. J Assoc Nurses AIDS Care 2014;25:603-13.

29. Huang D, Sangthong R, McNeil E, et al. Effects of a phone call intervention to promote adherence to antiretroviral therapy and quality of life of HIV/AIDS patients in Baoshan, China: a randomized controlled trial. AIDS Res Treat 2013;2013:1-9.

30. Shoukri MM, Asyali MH, Donner A. Sample size requirements for the design of reliability study: review and new results. Statistical Methods in Medical Research 2004;13:251-71.

31. CDC. 1993 revised classification system for HIV infection and expanded surveillance case definition for AIDS among adolescents and adults. MMWR Recomm Rep 1992;41:1-19.

32. CDC. Comparison of the revised World Health Organization and CDC surveillance case definitions and staging systems for HIV infection. MMWR Recomm Rep 2008;57:10-11.

33. Ware JE, Sherbourne CD. The MOS 36-item short-form health survey (SF-36). I. Conceptual framework and item selection. Med Care 1992;30:473-81.

34. Meng YJ, Li NX, Liu CJ, et al. Quality of life and hostile mentality trend of patients with HIV/AIDS in China. Public Health 2008;122:404-11. 
35. Bing EG, Hays RD, Jacobson LP, et al. Health-related quality of life among people with HIV disease: results from the Multicenter AIDS Cohort Study. Qual Life Res 2000;9:55-63.

36. Liu C, Ostrow D, Detels R, et al. Impacts of HIV infection and HAART use on quality of life. Qual Life Res 2006;15:941-9.

37. Zhu YX, Li T, Fan SR, et al. Health-related quality of life as measured with the Short-Form 36 (SF-36) questionnaire in patients with recurrent vulvovaginal candidiasis. Health Qual Life Outcomes 2016;14:1-6.

38. Hays RD, Sherbourne CD, Mazel RM. The RAND 36-Item Health Survey 1.0. Health Econ 1993;2:217-27.

39. Holmes WC, Shea JA. Performance of a new, HIV/AIDS-targeted quality of life (HAT-QoL) instrument in asymptomatic seropositive individuals. Qual Life Res 1997;6:561-71.

40. van Lummel RC, Walgaard S, Hobert MA, et al. Intra-rater, interrater and test-retest reliability of an Instrumented Timed Up and Go (iTUG) Test in patients with Parkinson's Disease. PLoS One 2016;11:e0151881.

41. Byrne BM. Structural equation modeling with Lisrel, Prelis, and Simplis. Basic concepts, applications, and programming. Structural Equation Modeling A Multidisciplinary Journal 1998;7:640-3.

42. Yekaninejad MS, Pakpour AH, Tadakamadla J, et al. Oral-healthrelated quality of life in patients with cancer: cultural adaptation and the psychometric testing of the Persian version of EORTC QLQOH17. Support Care Cancer 2015;23:1215-24.
43. Jiang XH, Shen ZZ, Zhang NN, et al. Research on reliability and validity of questionnaire. Mod Prev Med 2010;37:429-31.

44. Pereira M, Martins A, Alves S, et al. Assessing quality of life in middle-aged and older adults with HIV: psychometric testing of the WHOQOL-HIV-Bref. Qual Life Res 2014;23:2473-9.

45. Canavarro MC, Pereira M. Factor structure and psychometric properties of the european portuguese version of a questionnaire to assess quality of life in HIV-infected adults: the WHOQOL-HIV-Bref. AIDS Care 2012;24:799-807.

46. Tesfaye M, Olsen MF, Medhin G, et al. Adaptation and validation of the short version WHOQOL-HIV in Ethiopia. Int J Ment Health Syst 2016;10:29.

47. Chandra PS, Gandhi C, Satishchandra P, et al. Quality of life in HIV subtype $C$ infection among asymptomatic subjects and its association with CD4 counts and viral loads--a study from South India. Qual Life Res 2006;15:1597-605.

48. Peltzer K, Phaswana-Mafuya N. Health-related quality of life in a sample of HIV-infected South Africans. Afr J AIDS Res 2008;7:209-18.

49. Tran BX. Quality of life outcomes of antiretroviral treatment for HIV/ AIDS patients in Vietnam. PLoS One 2012;7:e41062. 